\title{
Visual search is guided by prospective and retrospective memory
}

\author{
Matthew S. Peterson, Melissa R. Beck, and Miroslava Vomela \\ George Mason University, Fairfax, Virginia
}

\begin{abstract}
Although there has been some controversy as to whether attention is guided by memory during visual search, recent findings have suggested that memory helps to prevent attention from needlessly reinspecting examined items. Until now, it has been assumed that some form of retrospective memory is responsible for keeping track of examined items and preventing revisitations. Alternatively, some form of prospective memory, such as strategic scanpath planning, could be responsible for guiding attention away from examined items. We used a new technique that allowed us to selectively prevent retrospective or prospective memory from contributing to search. We demonstrated that both retrospective and prospective memory guide attention during visual search.
\end{abstract}

When we search the environment, we often have some idea of what we are looking for, and through top-down guidance, attention is attracted to items that closely match the description of the search target (Treisman \& Gormican, 1988; Wolfe, 1994). Even without a goal in mind, attention does not flail around aimlessly. Instead, attention is attracted to the most salient visible items - namely, items that differ from other items in the environment, especially when they are different from their immediate neighbors (Wolfe, 1994; Wolfe \& Horowitz, 2004). That is, in the absence of top-down information, attention is driven by bottom-up information from the environment. How is attention guided when all bottom-up information is roughly identical (e.g., cases in which items that are nearly identical become indistinguishable when viewed in the periphery)? Some research has suggested that in the absence of bottom-up information that distinguishes among items, attentional deployments are random (Gibson, Li, Skow, Brown, \& Cooke, 2000; Horowitz \& Wolfe, $1998,2001)$. That is, when there is no salient information that can be used to discriminate one item from another, attention is deployed randomly, with no memory for which items have and have not been examined.

In contrast, other evidence has indicated that memory guides attention during search (Gilchrist \& Harvey, 2000; Hoffman \& Reiss, 2001; Kristjansson, 2000; McCarley, Wang, Kramer, Irwin, \& Peterson, 2003) and can prevent reexaminations for search sets of at least 12 items (Peterson, Kramer, Wang, Irwin, \& McCarley, 2001). What is unclear is the form of memory that is guiding attention. One possibility is that the mind keeps track of examined objects or locations (Klein \& MacInnes, 1999), a process that may be supplemented by such strategies as spatial chunking. Another possibility is that attention is guided by prospective, rather than retrospective, memory. This could involve planning a series of shifts to specific objects or something as mundane as searching in a clockwise pattern. This strategic planning could prevent revisitations, even in the absence of retrospective memory (Gilchrist \& Harvey, 2006; Zingale \& Kowler, 1987).

Until recently, the potential contribution of prospective memory to search had been largely ignored. Employing a novel task that prevented the planning of more than one saccade in advance, McCarley et al. (2003) measured the contribution of retrospective memory to visual search when the use of prospective memory was prevented. In their task, the observers were presented with only three items from the search set at any one time, with the display changing during each saccade. One of the items was always the currently fixated item, leaving two possible saccade targets. After several saccades, one of the saccade targets was always an old examined item, and the other was a new item. The subjects preferred making saccades to the new item and rarely revisited the last four items, suggesting that retrospective memory has a span of roughly four items.

If retrospective memory keeps track of only the last 4 items, why has other research, in which conventional static displays were used, demonstrated that items are rarely revisited when there are up to 12 items in the display? One possibility is that the lack of revisitations when static displays with 12 items are viewed might be due to prospective memories, such as scanpath planning or other types of strategies (Peterson et al., 2001). Another possibility is that the disappearance and reappearance of old examined items in the dynamic displays of McCarley et al. (2003) interfered with memory representations for examined items, leading to an underestimation of retrospective memory.

In the following series of experiments, we addressed the potential shortfall of the dynamic displays used in Mc- 
Carley et al. (2003) by employing a new technique that selectively leaves subsets of the search items static and visible throughout the search process (see Horowitz \& Wolfe, 1998 , for a related technique used for covert search, and Thornton \& Horowitz, 2004, for a related technique used with manual tracking). Leaving some of the items static gives any potential prospective or retrospective memory mechanisms a chance to generate a richer memory representation. We used a contingent oculomotor paradigm similar to that used by McCarley and colleagues, except that all the search items remained visible during a trial. During the course of a trial, selected subsets of items were moved to new locations during saccades, taking advantage of saccadic suppression to mask transients (Ross, Morrone, Goldberg, \& Burr, 2001). Using saccades to trigger display changes also prevents subjects from adopting a sit-and-wait strategy, which may have confounded the results from the dynamic displays of Horowitz and Wolfe (1998; see von Mühlenen, Müller, \& Müller, 2003, for a discussion). In Experiment 1, we examined the contributions of prospective memory by comparing a conventional static search condition with a condition in which the use of prospective memory was prevented. In Experiment 1, unexamined items were moved to new locations during each saccade, preventing use of prospective memory but allowing retrospective memory to track the static examined items. In Experiment 2, we examined the contribution of retrospective memory to the guiding of attention. In Experiment 2 , examined items were moved to a new location during each saccade, allowing prospective memory to plan ahead but preventing retrospective memory from tracking the old dynamic items (Peterson, Boot, Kramer, \& McCarley, 2004). In both experiments, if the dynamic condition disrupted the targeted memory process (retrospective or prospective), revisits should have increased in the dynamic condition, in comparison with the static condition. In contrast, if the dynamic conditions did not affect revisits, this would suggest that the targeted memory process was not used to guide search (Horowitz \& Wolfe, 1998).

By measuring observers' eye movements, we were able to track attention on a moment-by-moment basis. Eye movements and covert attention have been shown to be mandatorily coupled, with eye movements following the path of covert attentional shifts (Deubel \& Schneider, 1996; Hoffman \& Subramaniam, 1995; Kowler, Anderson, Dosher, \& Blaser, 1995; Peterson, Kramer, \& Irwin, 2004). Similarly, when the eyes are stable during a fixation, attention does not appear to scout out new locations but, instead, remains focused on the area being fixated, shifting only to the target of the next saccade once the saccade has been programmed (Peterson et al., 2001). Although it is certainly possible for individuals to move covert attention to an area in the periphery while the eyes are still, Peterson et al. (2001) found no evidence that subjects adopt this strategy while performing a search task. Instead, covert attention appears to be either centered at fixation or shifted to the next saccade destination. To prevent more than 1 item from being examined during a fixation, the items in both experiments were small and spaced far enough apart that each item had to be fixated individually (McCarley et al., 2003; Peterson et al., 2001). As a consequence, items were indistinguishable in peripheral vision, thereby minimizing bottom-up contributions to guidance. Any changes made to a display occurred during saccades, allowing saccadic suppression to mask any transients (Ross et al., 2001). The number of search items in the display was either 8 or 16 , bracketing set size 12 , which had previously demonstrated near-perfect memory with static displays (Peterson et al., 2001). In both experiments, trials were blocked into dynamic or static conditions.

\section{EXPERIMENT 1}

The goal of Experiment 1 was to examine the contribution of prospective memory by selectively removing the opportunity to plan more than one saccade in advance in an otherwise conventional visual search task. In Experiment 1 , examined items remained static, but unexamined items moved to new locations during each saccade.

\section{Method}

Subjects. Twelve George Mason University students participated in Experiment 1 ( 7 males and 5 females; mean age $=20.4$ years) for course credit.

Apparatus. A Power Macintosh G4 (Dual $1 \mathrm{GHz}$ ), equipped with a 20-in. (viewable) ViewSonic P225fb capable of running at $120 \mathrm{~Hz}$ at a resolution of $640 \times 480$, and running custom software, was used to present the stimuli, control the timing of experimental events, and record the subjects' response times. This computer was networked to a Dell Pentium 4 that collected eyetracking data in conjunction with an Eyelink 2 system (SR Research). Latency between the machines was $10 \mathrm{msec}$. The Eyelink 2 tracker (SR Research Ltd.) sampled at a rate of $250 \mathrm{~Hz}$ and had a spatial resolution of less than $0.5^{\circ}$. The Eyelink 2 used an infrared video-based tracking technology to compute the center and size of the pupils in both eyes and an infrared system to track head motion. Although head motion was measured, the head was stabilized by means of a chinrest located $57 \mathrm{~cm}$ from the monitor.

Design. Three independent variables were manipulated in a repeated measures orthogonal design: set size ( 8 or 16), display type (static or dynamic), and target presence (present or absent).

Stimuli. The stimuli consisted of white Ts and Ls approximately $0.19^{\circ}$ tall and $0.19^{\circ}$ wide $(3 \times 3$ pixels $)$ drawn on a black background. The minimum distance between the stimuli was $5^{\circ}$ ( 80 pixels). The possible locations where items could appear were arranged into eight columns and six rows, with the central four cells near the initial central fixation excluded. In addition, a programming error prevented the 3 rightmost locations in the bottom row from being chosen, limiting the number of possible locations to 41 . Item locations were chosen by randomly shuffling the list of 41 locations and picking the first 8 or 16 locations. In the dynamic conditions, the new locations were chosen by picking the next items in the randomized list. If the algorithm reached the end of the list, it started back from the bottom, excluding locations that were now assigned to static locations. This simple method ensured that several fixations passed before a location was reassigned to a dynamic item in the dynamic condition.

The targets were Ts rotated left or right of vertical. The distractors were normal or mirror-imaged Ls rotated $0^{\circ}, 90^{\circ}, 180^{\circ}$, or $270^{\circ}$. In the target-present trials, each display contained one target and 7 or 15 distractors (set sizes of 8 and 16, respectively). In the targetabsent trials, each display contained 8 or 16 distractors.

Procedure. The subjects fixated a central cross and pressed the space bar to start each trial. If the subjects fixated within $2^{\circ}$ of 
the cross, a drift correction occurred, and the trial proceeded. The search display was then presented. The subjects' task was to determine whether a target, a left or right $90^{\circ}$-rotated $\mathrm{T}$, was present in the display. The subjects responded by pressing the " $\mathrm{z}$ " or " " " key on the computer keyboard, and the mapping of the keys to target presence was counterbalanced across subjects. A tone sounded if an incorrect response was made.

In the dynamic condition, the locations of unexamined items changed during each saccade. The target of the current saccade was never moved. In the static condition, the locations of all the items remained static throughout each trial. To optimize detection speed, a method was used for online classification of saccades that was different from that used for offline data analysis. With the online method, a saccade was detected when its velocity exceeded 6 pixels $\left(0.38^{\circ}\right.$ of visual angle) during a single 4-msec sample, or approximately $95 \%$ sec. Two criteria were used to predict the landing point of each saccade so that a saccade target would not be moved. First, after the criterion for a saccade to be present had been exceeded, the velocity had to be less than 10 pixels $/ 4-\mathrm{msec}$ sample $(235 \% \mathrm{sec})$. The second criterion that had to be met was that the current eye position was within 36 pixels $\left(2.28^{\circ}\right)$ of an item. For data analysis, the offline method provided by Eyelink 2, which will be discussed at the beginning of the Results section, was used for saccade and fixation classification.

Each experimental session consisted of eight blocks of 32 trials, for a total of 256 trials during a single 45 -min session. The blocks consisted entirely of static or dynamic trials, presented in an ABAB design, with the order of presentation counterbalanced across subjects. The subjects were informed of the type of block before each block began.

\section{Results}

Manual responses. Manual responses more than three standard deviations from each cell's mean were excluded, as were response times (RTs) less than $200 \mathrm{msec}$. This led to the exclusion of approximately $1 \%$ of the trials. Manual RTs and accuracy can be seen in Table 1. The RTs and accuracy were analyzed in separate three-way repeated measures ANOVAs, with set size (8 or 16), display type (static or dynamic), and target presence (present or absent) as factors. The only effect for accuracy was a main effect of target presence, with responses slightly more accurate when the target was absent $[F(1,11)=5.0, p<.05]$. RTs were longer as the number of items in the display increased from 8 to $16[F(1,11)=37.7, p<.01]$, and RTs were longer when the target was absent $[F(1,11)=51.7, p<.01]$. In addition, RTs were longer in the dynamic condition than in the static condition $[F(1,11)=24.0, p<.01]$. As in previous visual search experiments, set size and target presence interacted, with responses slowing proportionally as the set size increased for the target-absent trials $[F(1,11)=23.3, p<.01]$. Display type interacted with set size, with responses in the dynamic condition having

Table 1

Mean Response Times (RTs, in Milliseconds) and Accuracy in Experiment 1

\begin{tabular}{llccccc}
\hline & & \multicolumn{2}{c}{ Set Size 8} & & \multicolumn{2}{c}{ Set Size 16 } \\
\cline { 3 - 4 } \cline { 6 - 7 } Display & Target & RT & Acc. & & RT & Acc. \\
\hline Static & present & 2,523 & .87 & & 3,751 & .88 \\
Static & absent & 3,576 & .89 & & 6,225 & .91 \\
Dynamic & present & 3,317 & .90 & & 5,323 & .91 \\
Dynamic & absent & 6,221 & .91 & & 10,818 & .94 \\
\hline
\end{tabular}

Table 2

Mean Numbers of Gazes and Gaze

Durations in Experiment 1

\begin{tabular}{cllcc}
\hline Set Size & Display & Target & Gazes & $\begin{array}{c}\text { Gaze } \\
\text { Duration } \\
(\mathrm{msec})\end{array}$ \\
\hline 8 & static & absent & 11.4 & 224.6 \\
8 & static & present & 9.5 & 221.4 \\
8 & dynamic & absent & 14.4 & 252.6 \\
8 & dynamic & present & 10.2 & 251.7 \\
16 & static & absent & 17.1 & 223.6 \\
16 & static & present & 12.1 & 219.6 \\
16 & dynamic & absent & 23.3 & 253.6 \\
16 & dynamic & present & 13.2 & 263.0 \\
\hline
\end{tabular}

an overadditive effect as set size increased $[F(1,11)=7.0$, $p<.05]$. The type of display also produced an overadditive effect, depending on whether the target was present or not $[F(1,11)=19.0, p<.01]$. The three-way interaction failed to reach significance $[F(1,11)=2.9]$, and an analysis of the search slopes showed that both the static and the dynamic conditions produced search slopes close to the 2:1 ratio expected for serial search (2.16 for static and 1.99 for dynamic).

Eye movements. For all the experiments, only saccades that landed on an item were counted as fixations, and consecutive fixations on the same item were considered a single gaze. Fixations were counted as landing on an item if they were within 36 pixels $\left(2.28^{\circ}\right)$ of the center of the item. Eye movements were classified as saccades if either (1) speed exceeded $30 \% \mathrm{sec}$ and acceleration exceeded $8,000^{\circ} / \mathrm{sec}^{2}$ or (2) acceleration exceeded $8,000^{\circ} / \mathrm{sec}^{2}$ and the distance exceeded $0.2^{\circ}$. Outliers were removed by excluding all trials on which manual RTs exceeded three standard deviations above the mean of each cell (approximately 1\%), and only trials with correct manual responses were included. All $t$ tests used Bonferronicorrected alphas.

The results for the mean number of gazes mirror those for the mean RTs. The number of gazes and mean gaze durations can be seen in Table 2. The number of gazes and mean gaze durations were analyzed in separate three-way repeated measures ANOVAs, with set size (8 or 16), display type (static or dynamic), and target presence (present or absent) as factors. More gazes occurred for set size 16 than for set size $8[F(1,11)=11.6, p<.01]$, and more gazes occurred when the target was absent than when it was present $[F(1,11)=16.9, p<.01]$. Dynamic trials also led to more gazes than did static trials $[F(1,11)=5.9$, $p<.05]$. As for the RTs, set size and target presence interacted, with the number of gazes for the target-absent trials increasing at a faster rate as set size increased $[F(1,11)=$ $26.1, p<.01]$. Also, as for the RTs, dynamic trials showed an increase in the number of gazes when the target was absent $[F(1,11)=6.9, p<.05]$. The three-way interaction failed to reach significance $[F(1,11)=3.9]$.

For the gaze durations, only type of trial had a significant effect on gaze duration, with dynamic trials producing longer gazes than did static trials $[F(1,11)=20.0, p<$ 
$.01 ; M \mathrm{~s}=222.3$ and 255.2 for static and dynamic trials, respectively].

Revisitation rates. Figure 1 shows the probability of choosing an old item as a function of gaze within a trial, broken down by set size and target presence. The probability of choosing an old item was always zero at the first gaze, since none of the other items had been fixated at this point within the trial. Likewise, the probability of choosing an old item at Gaze 2 was always zero, because all of the targetable items were new items; the only old item was the item the eyes had just left. As more items were examined, the likelihood that search would be terminated increased, leading to empty cells at later gazes. Only gazes with complete cells were included in the analyses.
For the target-present trials, only Gaze 6 showed a significant difference between the static and dynamic displays for set size $8[t(11)=3.60, p=.002]$. In contrast, for set size 16 , the subjects were less likely to reexamine an old item in the static condition than in the dynamic condition for nearly all gazes from 11 to $16[t(11)$, highest $p=.003]$. In the target-absent trials, the subjects were much less likely to reexamine an old item in the static condition than in the dynamic condition. Starting at the fourth (set size 8) or fifth (set size 16) gaze, gazes to old items were more likely to occur in the dynamic displays than in the static displays, and this trend held until the number of gazes approached the number of items in the display $[t(11)$, highest $p$ s $=.001$ and .002 for set sizes 8 and 16 , respectively].
Set Size 8, Present

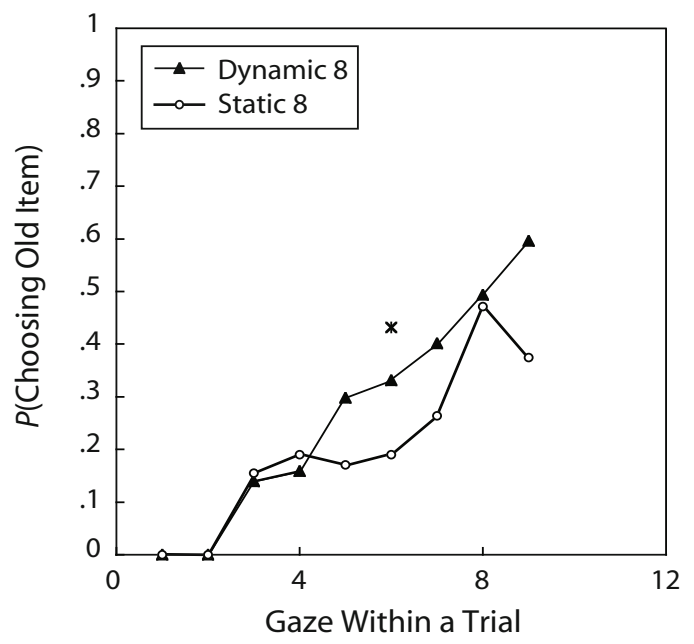

Set Size 8, Absent

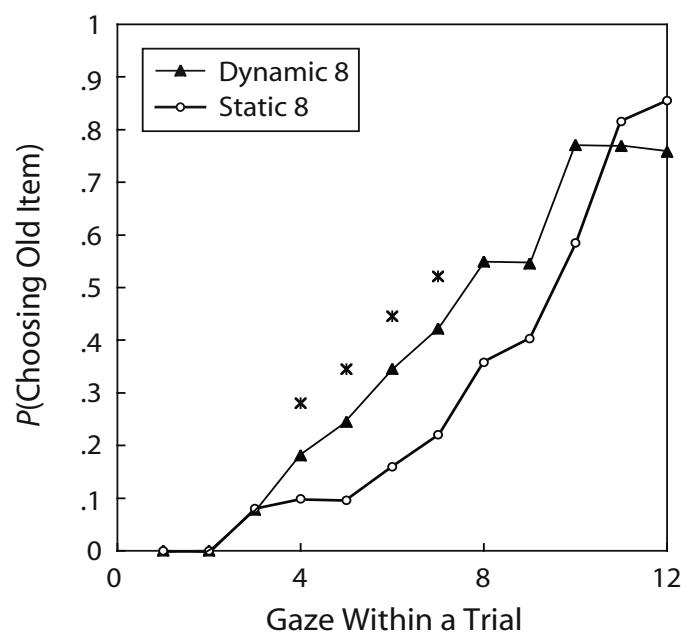

Set Size 16, Present

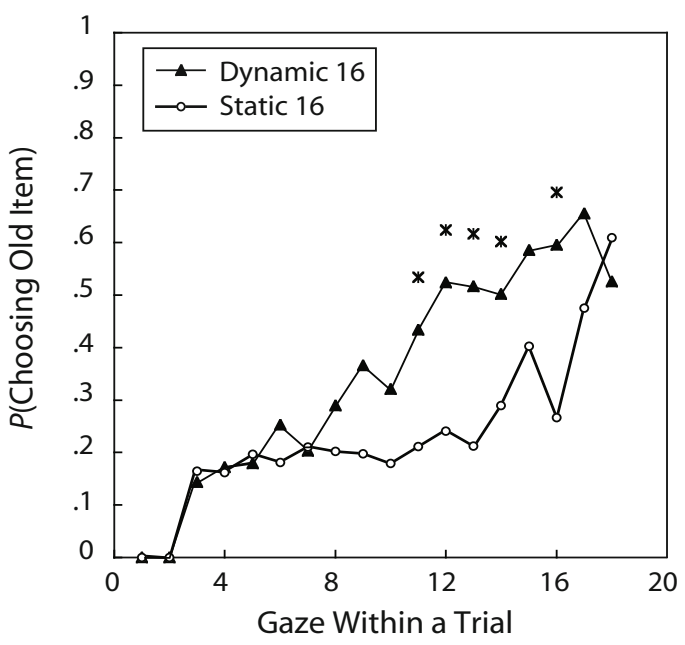

Set Size 16, Absent

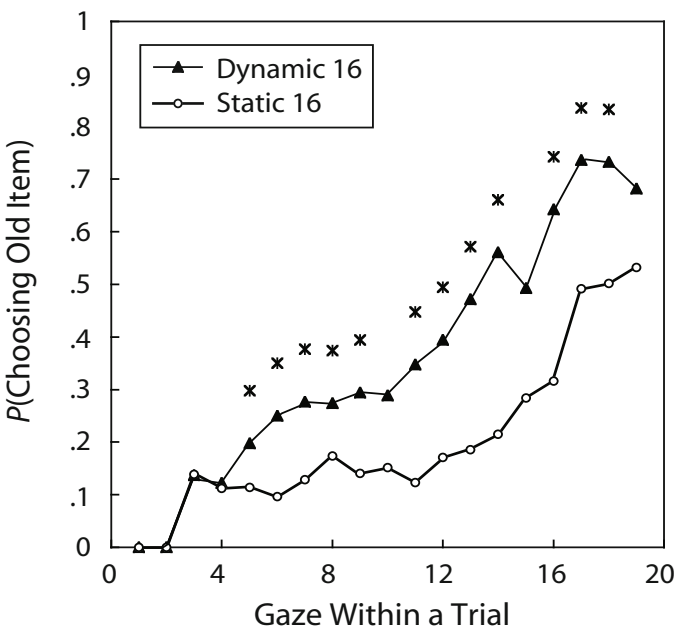

Figure 1. The probability of choosing an old item in Experiment 1 as a function of gaze within a trial. The left panels show the results for set size 8 , and the right panels for set size 16 . The top panels are for target-present trials, and the bottom panels are for target-absent trials. Open symbols are for trials in which the items remained static, and triangles represent trials in which the unexamined items moved to a new location during each saccade (dynamic). Stars represent significant differences between static and dynamic conditions. Data are not plotted for later gazes, due to sparse data. 
One point of interest is that the probability of refixating an old item jumped at the third gaze. If memory was slightly imperfect (Peterson et al., 2001), we might expect a more gradual rise in the revisitation rate, rather than the sudden jump. This jump at Gaze 3 seems to have been driven largely by saccades that returned to the last item fixated. Figure 2 shows the probability of revisiting an old item as a function of the number of gazes since it was last examined (lag), collapsed across target presence. As can be seen in Figure 2, a large number of the revisitations were made to the item that had been examined last (lag 2). Interestingly, reflexive saccades to the target accounted for roughly $25 \%$ of the lag 2 revisitations, suggesting that these were willful reexaminations to reinspect the last item (Peterson et al., 2001). Once again, the static displays showed a revisitation advantage over the dynamic displays, and this advantage held out to lag 14 for set size $16[t(11)$, highest $p \mathrm{~s}=.007$ and .0009 for set sizes 8 and 16 , respectively].

Figure 3 shows the probability of choosing an old item as a function of gaze within a trial, with lag 2 saccades removed. As in Figure 1, observers were more likely to examine old items when viewing the dynamic displays than when viewing the static displays, and this trend held until the number of gazes approached the number of items in the display $[t(11)$, highest $p \mathrm{~s}=.0056$ for set size 8 target present, .0015 for set size 16 target present, .0005 for set size 8 target absent, and .0017 for set size 16 target absent]. A series of $t$ tests comparing consecutive pairs of gazes in the set size 16 absent condition (this condition provides the situation in which the largest number of fixations and, therefore, the largest contribution of memory could be expected to occur) revealed that the probability of revisiting an old item did not significantly increase until the 17 th gaze $[t(11)=4.446, p=.0010]$.

Stereotypicality surface analyses. To examine whether the dynamic displays interfered with the stereotypicality of the subjects' scanpaths, we analyzed the order in which the different locations in the display were fixated. Because search displays were randomly generated on each trial, we chose a method that ignored individual configurations. We calculated the order in which the different screen locations had been examined. Although not all of the locations were used on every trial (more locations tended to be used on dynamic trials), the locations used were laid out in an $8 \times 6$ grid, allowing some commonality across trials. The four locations around central fixation were never used (due to a programming error, the three in the lower right corner also were never used). Because of missing data in some cells, we decided to collapse across target-present and target-absent conditions and limit the analysis to set size $16 .{ }^{1}$

Figure 4 shows the mean order in which different screen locations were visited for the static and dynamic conditions of set size 16, collapsed across target presence. To calculate the degree of stereotypicality, using multidimensional regression, we fitted 2-D surfaces to each subject's data and calculated the individual slants (i.e., slopes; 2 subjects were excluded due to empty cells). High average slants indicate more stereotypical search patterns, and if the subjects show little stereotypicality, the average slant should be near zero. The average surface slant for the static condition was $30.19^{\circ}$ and was significantly different from the mean slant for the dynamic condition $[M=$ $\left.21.36^{\circ} ; t(9)=8.84, p<.05\right]$.

\section{Discussion}

Performance in the dynamic conditions suffered, in comparison with the static conditions: Responses were slower overall, and more gazes were required to complete the search, suggesting that the subjects benefited from the opportunity to plan future attentional deployments in the static condition. Of particular note is the increase in gaze duration in the dynamic condition. One possibility is that the location changes in the dynamic displays interfered with targeting by changing the scene context. This possibility will be discussed and dismissed as unlikely in the Discussion section for Experiment 2. Another possibility

\section{Set Size 8}

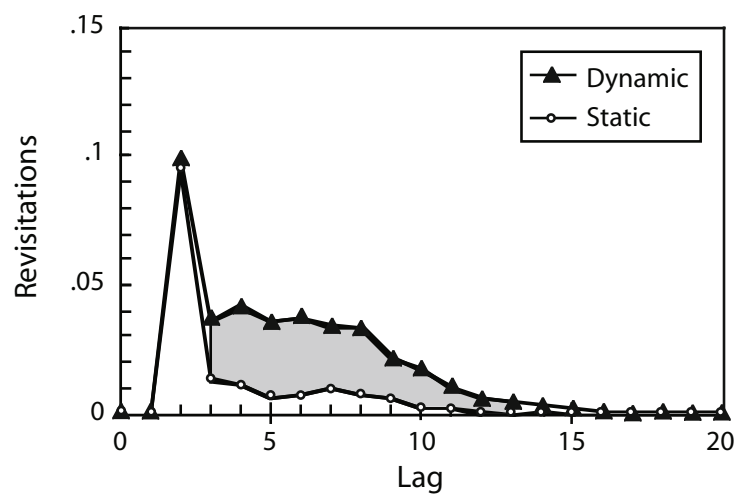

Set Size 16

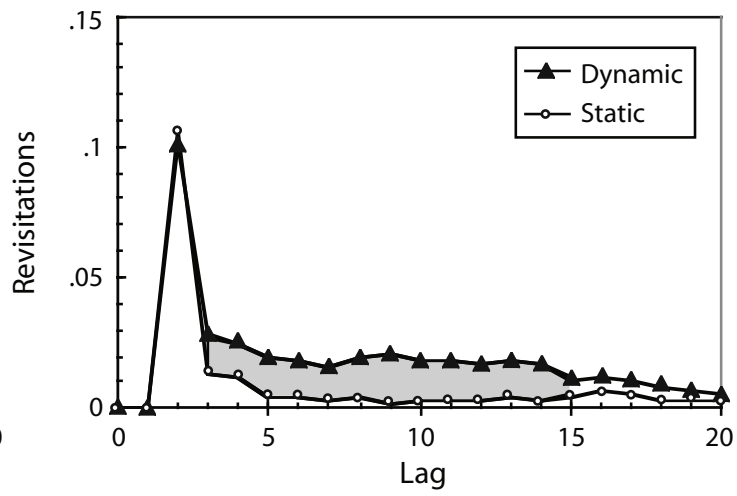

Figure 2. The probability of reexamining an item in Experiment 1 as a function of the number of intervening gazes (lag), collapsed across target presence. The left panel shows the results for set size 8, and the right panel for set size 16. Open symbols are for trials on which the items remained static, and triangles represent trials on which the unexamined items moved to a new location during each saccade (dynamic). The shaded area shows significant differences between static and dynamic conditions. 

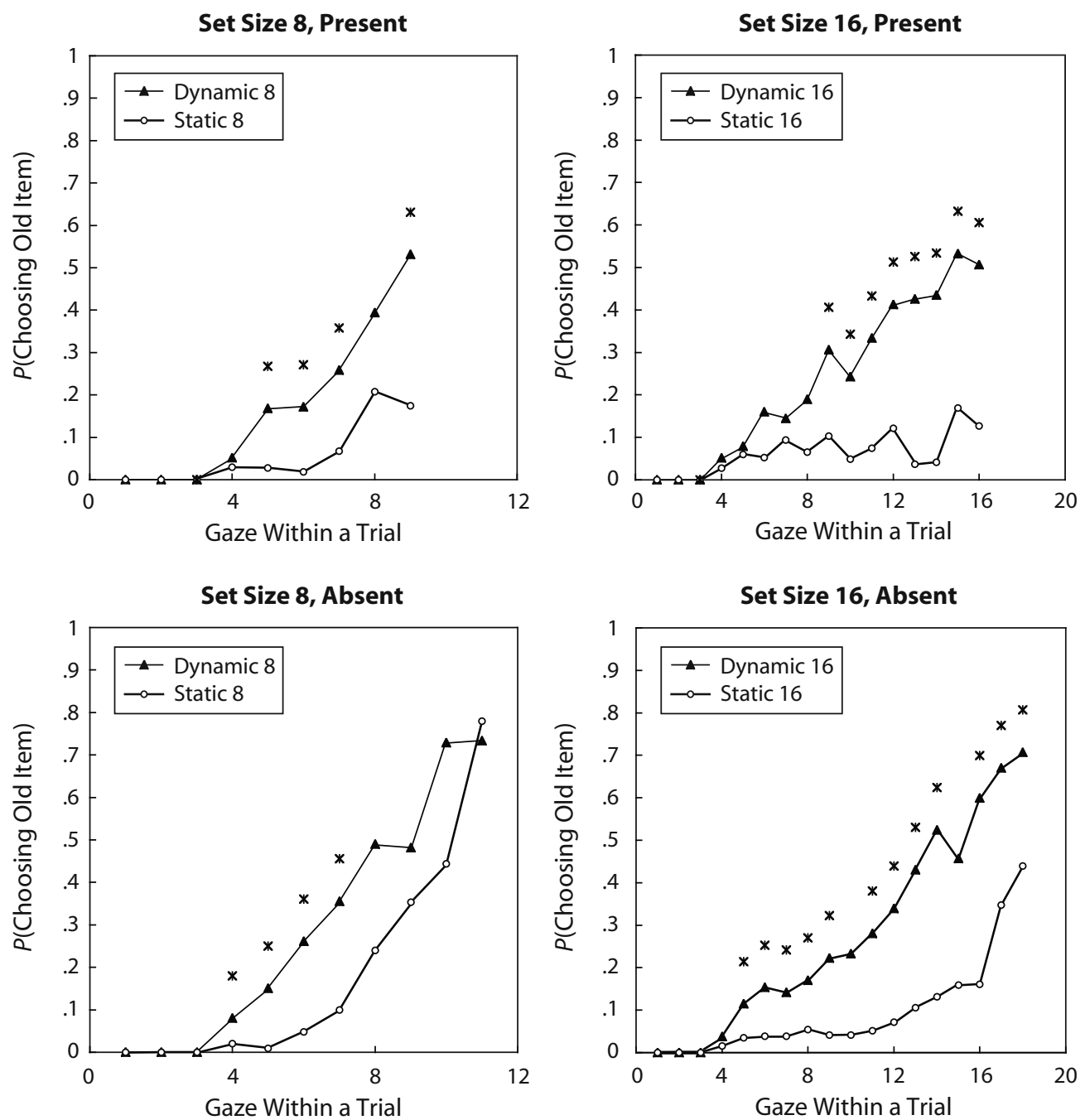

Figure 3. The probability of choosing an old item in Experiment 1 as a function of gaze within a trial, with lag 2 saccades removed. The left panel shows the results for set size 8 , and the right panel for set size 16 . The top panels are for target-present trials, and the bottom panels are for target-absent trials. Open symbols are for trials in which the items remained static, and triangles represent trials in which the unexamined items moved to a new location during each saccade (dynamic). Stars represent significant differences between static and dynamic conditions.

is that changing the locations of the unexamined items interfered with future planning. This implies that the saccade to the next item is planned during an earlier gaze, rather than during the current gaze.

An examination of the revisitation data supports the hypothesis that people rely on prospective memory while performing visual search. When the subjects were denied the opportunity to use prospective memory in the dynamic conditions, they were more likely to reexamine an item than they were in the static conditions. This trend was not as strong in the target-present conditions, due to the large number of saccades returning to the last examined item, which disproportionately consisted of the target (see also Peterson et al., 2001). These effects can be clearly seen by examining the static conditions of set size 16 in Figure 1. When the target was present, revisitations occurred almost $20 \%$ of the time during Gazes 3-12, whereas when the tar- get was absent, return gazes were reduced to almost $10 \%$. These return saccades suggest that individuals sometimes examined an item, made a saccade to the next item before a decision was made, and made a return saccade to reexamine the item. This implies that in normal search, the decision about which item to examine next is often made prior to the fixation immediately preceding the saccade (Zingale \& Kowler, 1987).

Because there were no targets to reexamine in the targetabsent trials, target-absent trials provided a purer measure of memory for distractors. For both set sizes, revisitation rates in the static conditions were markedly lower than those in the dynamic conditions, and this trend continued until the number of gazes approached the number of items in the display. These effects were even more pronounced when return saccades to the last examined item were removed (Figure 3 ). The probability of revisiting an 


\section{Static}

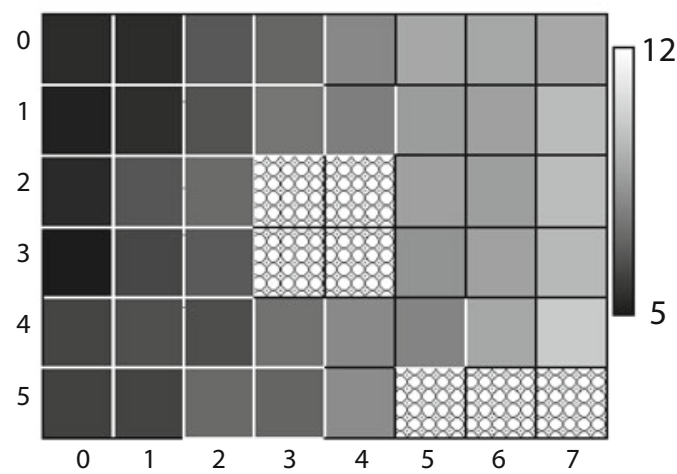

Dynamic

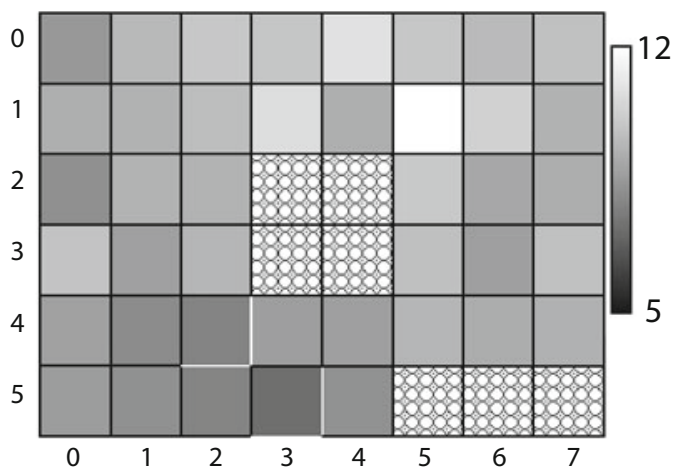

Figure 4. The mean order in which locations in the display were visited in Experiment 1, collapsed across target-present and target-absent trials for set size 16. The left panel shows the results for the static condition, and the right panel shows the results for the dynamic condition. Dark squares indicate areas that were visited early in a trial, and lighter squares indicate locations that were visited later in a trial. Patterned areas indicate areas in which stimuli could not appear.

item remained low and increased only as the number of gazes approached the number of items in the display. The revisitations at later gazes were likely due to the subjects' double-checking the display when a target had not been found. Indeed, at the largest set size, the probability of revisiting an old item did not significantly increase until the 17 th item.

Another way to examine potential effects of prospective memory is to examine the stereotypicality of the scanpaths. Although each display was randomly generated, we might expect a preferential search strategy to show up as a bias in the order in which different regions of the display were examined. If this bias was limited to choosing the next item to examine, the dynamic display should have had no effect on the order in which regions were visited over the course of an experiment, since the next item that was examined remained stable in the dynamic condition (i.e., saccade targets were never moved). In contrast, if this bias was the result of planning several saccades in advance, the dynamic condition should disrupt this plan, leading to a weaker observed bias than in the static condition. As can be seen in Figure 4, the dynamic condition clearly shows a weaker bias than does the static condition, suggesting that the bias observed in the static condition was due to some form of long-range planning.

Taken as a whole, these results suggest that when objects in the environment are indistinguishable, prospective memory plays a large role in guiding attention to items for closer examination. That is, subjects are able to search somewhat efficiently even when the only information available to guide attention is the locations of unexamined items.

\section{EXPERIMENT 2}

The goal of Experiment 2 was to examine the contribution of retrospective memory by selectively removing the opportunity to keep track of examined items in an otherwise conventional visual search task. Experiment 2 was identical to Experiment 1, except that unexamined items remained static throughout a trial and examined items randomly moved to new locations during saccades.

\section{Method}

Subjects. Twelve subjects participated in Experiment 2 (2 males and 9 females; mean age $=19.7$ years) for course credit.

\section{Results}

Manual responses. Manual RTs and accuracy are shown in Table 3. As in Experiment 1, RTs increased as set size increased $[F(1,11)=111.9, p<.01]$, RTs were longer in the dynamic condition $[F(1,11)=5.9, p<$ $.05]$, and RTs were shorter when the target was present $[F(1,11)=86.1, p<.01]$. Set size and target presence interacted, with set size having a larger effect when the target was absent $[F(1,11)=34.6, p<.01]$. As in Experiment 1 , type of display also produced an overadditive effect, depending on whether the target was present or not $[F(1,11)=6.4, p<.05]$. Set size, target presence, and display type interacted $[F(1,11)=7.4, p<.05]$, with only the static condition producing search slopes that were close to the 2:1 ratio expected for serial search (2.24 for static and 1.35 for dynamic). None of the other effects reached significance.

Manual responses were more accurate for the smaller set size $[F(1,11)=49.1, p<.01]$, for the static displays $[F(1,11)=36.2, p<.01]$, and when the target was absent $[F(1,11)=77.9, p<.01]$. Set size and display type inter-

Table 3

Mean Response Times (RTs, in Milliseconds) and Accuracy in Experiment 2

\begin{tabular}{llrrrrr}
\hline & & \multicolumn{2}{c}{ Set Size 8} & & \multicolumn{2}{c}{ Set Size 16} \\
\cline { 3 - 4 } \cline { 7 - 7 } Display & Target & RT & Acc. & & RT & Acc. \\
\hline Static & present & 2,488 & .99 & & 3,528 & .93 \\
Static & absent & 3,408 & 1.00 & & 5,674 & .96 \\
Dynamic & present & 2,610 & .95 & & 3,708 & .88 \\
Dynamic & absent & 4,826 & .98 & & 6,360 & .93 \\
\hline
\end{tabular}


acted, with accuracy decreasing more rapidly for the dynamic condition as set size increased $[F(1,11)=7.1, p<$ $.05]$. Similarly, target presence interacted with set size, with accuracy decreasing more rapidly when a target was present as the set size increased $[F(1,11)=7.3, p<.05]$. Display type also interacted with target presence, with the presence of a target decreasing accuracy more in the dynamic condition $[F(1,11)=14.7, p<.05]$. The three-way interaction failed to reach significance $(F=0.436)$.

Eye movements. The number of gazes and mean gaze duration are shown in Table 4. The number of gazes and mean gaze duration were analyzed in separate three-way repeated measures ANOVAs, with set size (8 or 16), display type (static or dynamic), and target presence (present or absent) as factors. More gazes occurred in set size 16 than in set size $8[F(1,11)=178.1, p<.01]$, and more gazes occurred when the target was present than when it was absent $[F(1,11)=94.1, p<.01]$. As in Experiment 1 , dynamic trials also led to more gazes than did static trials $[F(1,11)=5.1, p<.05]$. As for RTs and as in Experiment 1 , set size and target presence interacted, with the number of gazes for the target-absent trials increasing at a faster rate as set size increased $[F(1,11)=87.4, p<.01]$. None of the other interactions reached significance.

For the gaze durations, only the presence of a target had an effect on gaze duration, with target-absent trials producing slightly longer mean gaze durations $[F(1,11)=20.0$, $p<.01 ; M \mathrm{~s}=227.5$ and 216.8 for absent and present, respectively]. Unlike in Experiment 1, type of display had no effect on gaze durations $[F(1,11)=3.4, p>.05 ; M \mathrm{~s}=$ 218.5 and 225.3 for static and dynamic, respectively].

Revisitation rates. Figure 5 shows the probability of choosing an examined item as a function of gaze within a trial. As in Experiment 1, the first two gazes would never be on an old item, since all of the targetable items were considered new. As in Experiment 1, due to the larger number of observations at each gaze, the target-absent trials showed a larger effect (unlike with target-present trials, the subjects were unlikely to terminate search in the targetabsent trials until almost all items had been examined). In addition, because of the opportunity to reexamine targets, the probability of revisiting an item was greater in the target-present static conditions than in the target-absent static conditions. The static condition shows the same general pattern as that in Experiment 1, with the rate of revisi-

Table 4

Mean Numbers of Gazes and Gaze Durations in Experiment 2

\begin{tabular}{cllcc}
\hline Set Size & Display & Target & Gazes & $\begin{array}{c}\text { Gaze } \\
\text { Duration } \\
(\mathrm{msec})\end{array}$ \\
\hline 8 & static & absent & 7.5 & 221.3 \\
8 & static & present & 4.9 & 211.4 \\
8 & dynamic & absent & 10.2 & 230.4 \\
8 & dynamic & present & 6.1 & 218.1 \\
16 & static & absent & 15.4 & 222.4 \\
16 & static & present & 8.9 & 212.0 \\
16 & dynamic & absent & 16.8 & 231.0 \\
16 & dynamic & present & 9.8 & 216.1 \\
\hline
\end{tabular}

tations accelerating in the static conditions as the number of gazes approached the number of items in the display. Starting at the 4th (set size 8 ) and 11th (set size 16) gazes in the target-absent conditions, more revisitations occurred in the dynamic than in the static conditions [ $t(11)$, highest $p \mathrm{~s}=.0009$ and .0013 for set sizes 8 and 16, respectively]. This effect was less evident in the target-present conditions, with the static condition showing less revisitations than did the dynamic condition only for the 7th gaze for set size 8 and only for Gazes $13-15$ and 17 for set size 16 $[t(11)$, highest $p s=.0009$ and .0055 for set sizes 8 and 16 , respectively].

Stereotypicality surface analyses. Figure 6 shows the mean order in which different screen locations were visited for the static and dynamic conditions of set size 16 , collapsed across target presence. The data from 2 subjects were excluded, due to empty cells. The average surface slant for the static condition was $51.54^{\circ}$ and was significantly different from the mean slant for the dynamic condition $\left[M=40.37^{\circ} ; t(9)=5.53, p<.01\right]$.

\section{Discussion}

As in Experiment 1, performance in the dynamic condition suffered, in comparison with the static condition, with slower responses ${ }^{2}$ and more gazes required to complete the search. Unlike in Experiment 1, there was no effect on gaze duration in the dynamic condition. One possibility for the increased gaze durations in Experiment 1 was that the constantly changing scene context interfered in some manner. However, the lack of an effect on gaze duration in Experiment 2 (where changes in scene context also occurred) makes this possibility unlikely. Since dynamic displays led to increased gaze durations only when the unexamined items were moved to new locations, the increased gaze durations in Experiment 1 suggest that moving the new items interfered with planning future saccades. In contrast, new items remained static in Experiment 2, allowing the subjects to plan more than one saccade in advance.

Unlike in Experiment 1, revisitations were delayed in the dynamic conditions until later gazes, at least for set size 16. This suggests that in the dynamic condition, the subjects were able to take advantage of prospective memory to plan several saccades in advance, and revisitations increased only when the span of prospective memory had been surpassed. Support for this comes from the stereotypicality analysis. Although scanpaths were more stereotypical in the static condition, as can be seen in Figure 6, the difference was quite small. However, this revisitation effect did not hold for set size 8 . In the target-absent condition, which provides a more powerful test than do)es the target-present condition, revisitations were significantly greater in the dynamic condition than in the static condition, beginning with the fourth gaze. One possibility is that the memory representation used by prospective memory is spatial, rather than object based. If prospective memory used a coarse spatial representation, the greater spatial density inherent in the larger set size could have allowed for more items to fall within a spatial region, effectively 
Set Size 8, Present

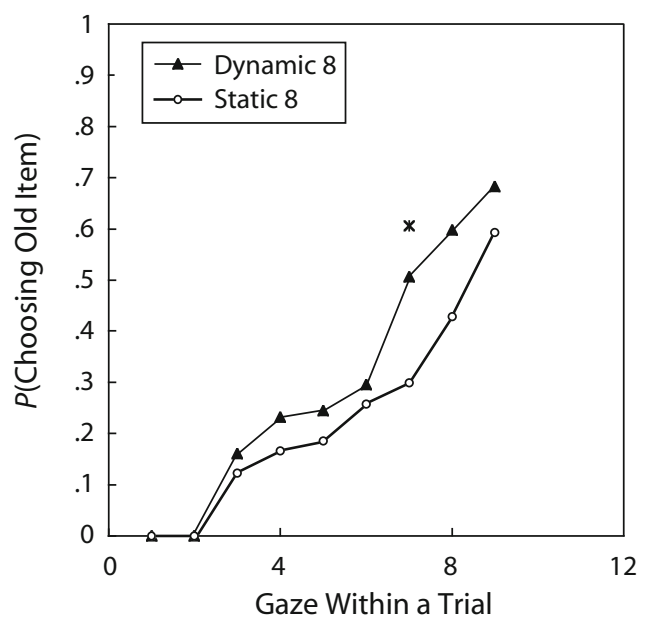

Set Size 8, Absent

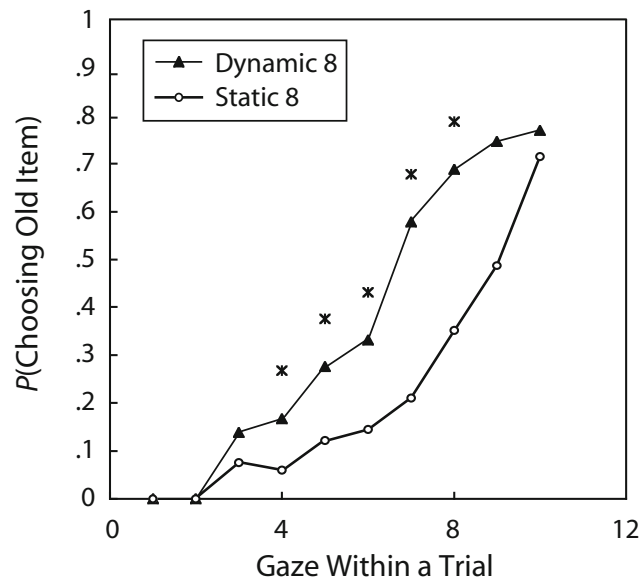

Set Size 16, Present

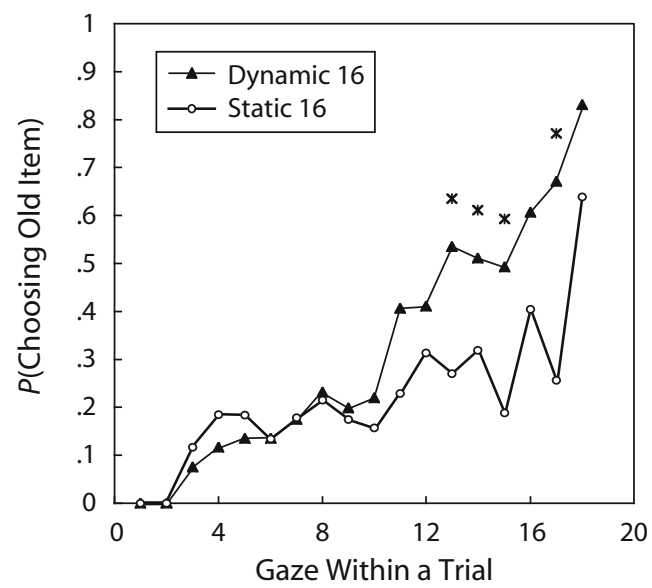

Set Size 16, Absent

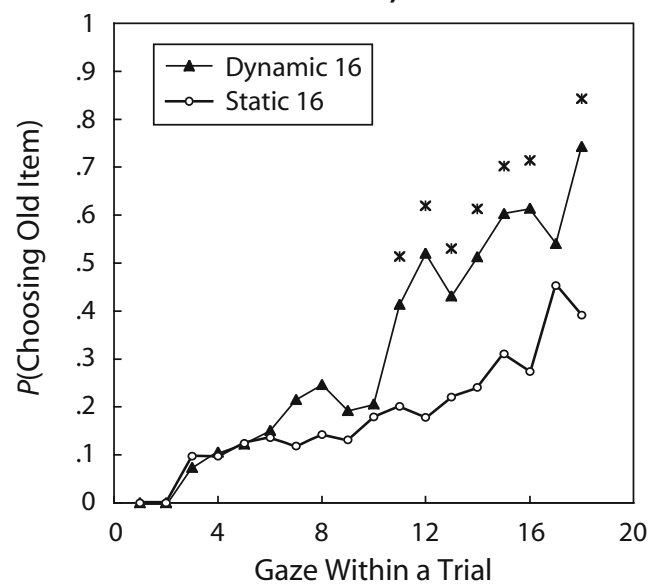

Figure 5. The probability of choosing an old item in Experiment 2 as a function of gaze within a trial. The left panel shows the results for set size 8, and the right panel for set size 16. The top panels are for target-present trials, and the bottom panels are for target-absent trials. Open symbols are for trials on which the items remained static, and triangles represent trials on which the unexamined items moved to a new location during each saccade (dynamic). Stars represent significant differences between static and dynamic conditions.

working as a form of chunking (Boot, Kramer, McCarley, Peterson, \& Scialfa, 2004) and leading to benefits as density increased due to increasing set size.

\section{GENERAL DISCUSSION}

Taken as a whole, the results of Experiments 1 and 2 indicate that both prospective and retrospective memory play a role in guiding visual search. Previous research in which static displays were used suggested that people have a memory for up to 12 items (Peterson et al., 2001). In contrast, when McCarley et al. (2003) used a task that prevented the use of prospective memory, only the last 4 items or so were avoided. This finding suggested that the earlier results with static displays had been inflated by prospective memory.

The experiments reported here were similar to the dynamic displays used by McCarley et al. (2003), but pro- vided a richer context for memory by holding a subset of items static and allowing them to remain visible throughout a trial. In contrast, the oculomotor contingent task in McCarley et al. displayed only a subset of search items during a single fixation. Because items were constantly disappearing and reappearing, that method might have underestimated the contribution of retrospective memory. In Experiment 1, once an item had been examined, it remained static throughout the remainder of the trial. In contrast, items that had not yet been examined moved to new locations during each saccade, preventing prospective memory from effectively planning ahead. As in previous research, we found that the majority of revisitations were made to the item that had just been examined (Motter \& Belky, 1998) and that a substantial proportion of these lag 2 revisits $(25 \%)$ occurred when the item was the target (Peterson et al., 2001), and this trend held for both the static and the dynamic conditions. Because a large pro- 

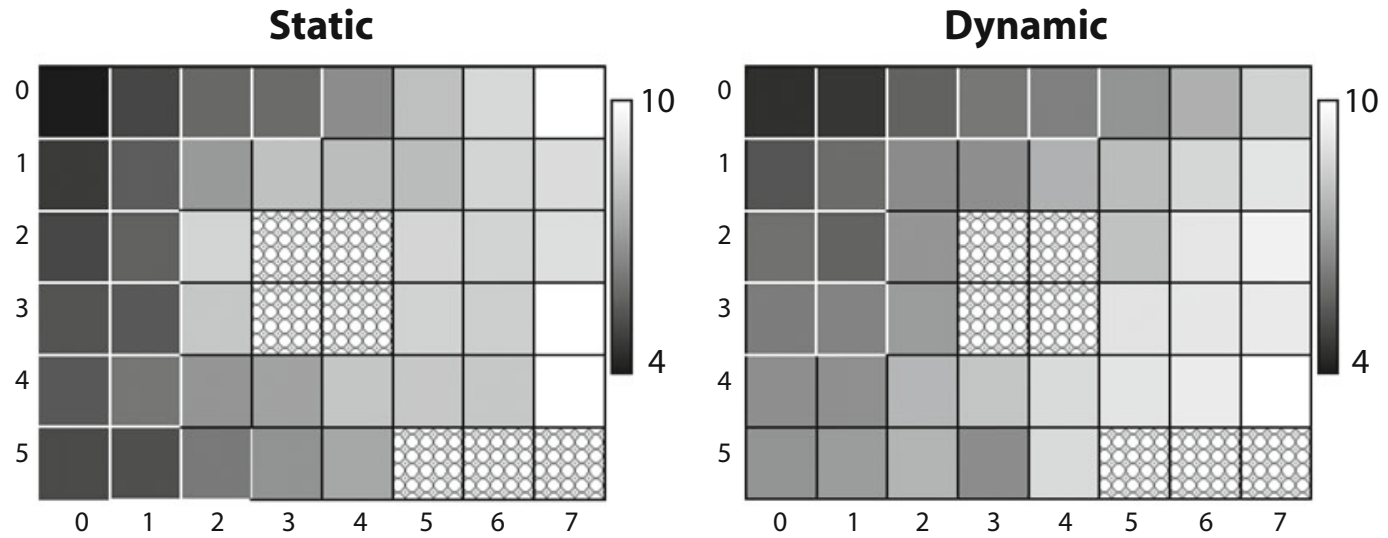

Figure 6. The mean order in which locations in the display were visited in Experiment 2, collapsed across target-present and target-absent trials for set size 16. The left panel shows the results for the static condition, and the right panel shows the results for the dynamic condition. Dark squares indicate areas that were visited early in a trial, and lighter squares indicate locations that were visited later in a trial. Patterned areas indicate areas in which stimuli could not appear.

portion of these lag 2 revisits were to the target, this suggests that these lag 2 revisits were willful reexaminations of items that had been inadequately examined before the next saccade was launched.

Removing these lag 2 revisitations reveals a substantial deficit in the dynamic condition, in comparison with the static. This deficit starts at roughly the 4th or 5th gaze and continues up to the 18th gaze (set size 16 target absent). This suggests that in the dynamic condition, performance is limited by the capacity of retrospective memory, whereas the static condition can rely on prospective memory to prevent revisitations. This suggests that retrospective memory keeps track of only the last few items and is in line with previous findings using the contingent oculomotor (McCarley et al., 2003) and the inhibition of return (IOR; Klein \& MacInnes, 1999) paradigms.

Experiment 2 tested the ability of prospective memory to prevent revisits and lead to efficient search by moving examined items to new locations during saccades. Strong support for the role of prospective memory comes in the form of the stereotypicality analyses. In the first experiment, moving the new items led to a flattening of the stereotypicality surface, in comparison with the corresponding static condition. In contrast, moving the old items in Experiment 2 had only a minor effect on the stereotypicality surface, in comparison with the static condition. The increased gaze duration in Experiment 1, in which new items were moved, suggests that saccade planning is not limited to the next item. More specifically, in Experiment 1 , the target of the saccade was never moved. This suggests that the increased gaze durations were due to the dynamic displays' interfering with the ability to plan two or more future saccades (Gilchrist \& Harvey, 2006; Zingale \& Kowler, 1987).

Interestingly, in Experiment 2, revisitations in the dynamic condition occurred later in the search for set size 16 than in the search for set size 8 . This suggests that in the dynamic condition, in which examined items moved during saccades, the subjects were able to take advantage of prospective memory to prevent items from being needlessly reexamined with set size 16 . Why was performance so poor for the smaller set size? If prospective memory has a fixed capacity of $n$ items, performance in the dynamic condition should have been much better for the smaller set size than for the larger set size. One possibility is that prospective memory does not plan saccades to individual items but, instead, plans saccades to regions of space. More items are likely to be found in a given region with the larger set size than with the smaller set size, and this spatial chunking might account for the superior performance in the dynamic condition for the larger set size. Although the experiments reported here were not designed to test this chunking hypothesis, aging research in which the oculomotor contingent task in McCarley et al. (2003) has been used has suggested that retrospective memory might encode spatial regions, rather than individual objects (Boot, Kramer, et al., 2004).

One argument against the results reported here is that moving unexamined or examined items to new locations may not have selectively disrupted prospective or retrospective memory while leaving the other memory process unaffected. For example, moving unexamined items to new locations led to a constantly shifting scene context, and the changing context might have interfered with a retrospective representation. There is some evidence that memory for the spatial location of an object is affected by the global consistency of a scene between encoding and testing (Jiang, Olson, \& Chun, 2000). However, this may not hold across eye movements, since transsaccadic memory is limited to roughly four items and is strongest for items that are the target of the saccade (Henderson \& Hollingworth, 1999; Irwin \& Gordon, 1998).

Likewise, findings from the contextual-cuing literature bring a mixed message in regard to the role of scene context within a single trial. Minor distortions to configurations on each exposure of a repeated configuration, such 
as randomly moving a single item (out of 12) to a new location, have been shown to have no effect on learning scene contexts, in comparison with configurations in which all of the items remained static from exposure to exposure (Peterson \& Kramer, 2001). Similarly, training subjects to associate a target location with two different displays and later recombining the two displays to form a novel display has been shown to lead to perfect transfer of contextual cuing (Jiang \& Wagner, 2004). In addition, contextual cuing by scene contexts tends to take several exposures, making it unlikely that the individuals in Experiment 1 learned the scene context over the course of a trial (Chun \& Jiang, 1998). Likewise, the contextualcuing effect, as measured by RTs, appears to be driven largely by a small number of trials (Peterson \& Kramer, 2001). In that study, the proportion of trials in which the eyes went directly to the target increased from $7.1 \%$ in the random displays to $11.3 \%$ in the repeated displays (with 12 items in the display, the chance level of first looking at the target was $8.3 \%$ ). This suggests that contextual cuing is the exception, rather than the rule. Finally, although the addition of static landmarks to an oculomotor contingent task identical to that used in McCarley et al. (2003) has been shown to improve retrospective memory, the overall effect has been quite small ( $1 \%-2 \%$ less likely to revisit an old item, with chance at $50 \%$ ), suggesting that moving the unexamined items in Experiment 1 likely had a negligible effect on retrospective memory (Peterson, Boot, et al., 2004). Nevertheless, any effect of changing the scene context would have been to minimize the contribution of the nontargeted memory and, in turn, to lead to an underestimate of the nontargeted memories in Experiments 1 and 2 .

Previous research in which search through static displays has been contrasted with search through dynamic displays has shown no difference in performance between static and dynamic displays (Horowitz \& Wolfe, 1998). Since search through the static displays was no better than search through the dynamic displays, it was concluded that there was no memory available to guide visual search. In contrast, we found clear evidence for memory. The two experiments reported here differ from previous work in two important ways. First, our dynamic displays were saccade contingent, whereas the displays used by Horowitz and Wolfe (1998) automatically changed every $100 \mathrm{msec}$. The automatically changing displays could have led the subjects to adopt a sit-and-wait strategy (von Mühlenen et al., 2003), thereby eliminating the need to use memory to guide visual search. However, our saccade-contingent displays prevented the use of a sit-and-wait strategy. The second manner in which these displays differed is that our stimuli were small enough that they prevented parallel search (Klein, Shore, MacInnes, Matheson, \& Christie, 1998) or parallel guidance (Wolfe, 1994) from taking place.

The inability to use covert attention to guide search (due to the lack of peripheral feature information for the small items used in the search displays) may appear to limit the generalizability of our results. For example, searches that can be performed at fast rates with covert attention may not be guided by memory, whereas searches that are performed at slow rates and require eye movements may be guided by memory. However, interpreting fast searches as searches that necessarily involve rapid shifts appears to be a misinterpretation of RT search slopes. For example, since covert attention appears to be able to shift every $200-500 \mathrm{msec}$ (Horowitz, Holcombe, Wolfe, Arsenio, \& DiMase, 2004; Theeuwes, Godijn, \& Pratt, 2004), how do we account for search slopes that fall into the $10-200 \mathrm{msec} /$ item range? The simple answer for this comes in the form of attentional guidance. An extreme example of this is pop-out, in which the time to find a unique target is nearly independent of search set size. When pop-out occurs, attention is guided immediately to the target, and the target is the only item examined. Search slopes in this example are nearly zero, not because of extremely rapid shifts, but because of the lack of variability in the number of shifts for each set size (Findlay, 1997). Intermediate search slopes can be explained by strong or weak guidance, with guidance limiting the number of potential candidates on the basis of their physical features and how closely they match a top-down attentional set (Wolfe, 1994) and, hence, the number of saccades (Motter \& Belky, 1998; Zelinksy \& Sheinberg, 1997). Given that it appears that fast searches are not due to shifts of attention that are qualitatively different from those involved in slower searches, it is unreasonable to suspect that efficient searches are guided by memory any less than are slow searches.

Although several studies have confirmed that attention is guided by memory, what has been unclear is the form of memory used to guide attention (Klein \& MacInnes, 1999; Kristjansson, 2000; McCarley et al., 2003; Peterson et al., 2001). The results of Experiments 1 and 2 suggest that attention is guided by both retrospective and prospective memory mechanisms. In Experiment 1, moving the unexamined items to new locations interfered with search, demonstrating that prospective memory is a large component in guiding attention during search. In Experiment 2, we found further evidence that retrospective memory guides attention, but this was partially masked by the strong effect of prospective memory, particularly in the larger set size.

What sorts of memory representations might underlie search? Retrospective memory during search appears to be largely automatic, since observers find it difficult to refixate visited items when explicitly told to do so (Boot, McCarley, Kramer, \& Peterson, 2004). One possibility is that this automatic component is IOR, which involves a spatial representation of previously inspected locations in the parietal region (Sapir, Hayes, Henik, Danziger, \& Rafal, 2004). Support for this has come from the dualtask literature, which has shown that concurrent spatial working memory tasks, which also involve the parietal area, interfere with IOR and with visual search (Castel, Pratt, \& Craik, 2003; Oh \& Kim, 2004; Woodman \& Luck, 2004). Given the results of Experiment 2 and the fact that spatial chunking has been observed for retrospective memory during search (Boot, Kramer, et al., 2004), it can be suggested that both retrospective and 
prospective memory may use a spatial representation involving dorsal stream, rather than ventral stream, object representations (Irwin \& Brockmole, 2004). Interestingly, spatial-scaling effects during search appear to change as individuals age, with older adults showing broader representations (Boot, Kramer, et al., 2004; Greenwood \& Parasuraman, 2004).

Although retrospective and prospective memory may use the same memory representation, it is likely that they are driven by different mechanisms. For example, IOR appears to be an automatic process that is immune from interference from concurrent working memory tasks, such as adding digits or other tasks that do not use a spatial representation (Castel et al., 2003). On the other hand, tasks that stress the central executive (e.g., counting or sorting a string of letters alphabetically) do interfere with visual search, even when the representation used by the working memory task is not spatial in nature (Han \& Kim, 2004). One possibility is that prospective memory is nonautomatic and requires the central executive for conscious planning of which items to examine in the future. Thus, when the central executive is taxed, prospective memory is unable to help guide attention during search, leaving retrospective memory as the mechanism by which items are prevented from being needlessly reexamined.

\section{AUTHOR NOTE}

This work was supported by National Institutes of Health Grant R01 MH64505 to M.S.P. We thank Steve Beretzky for his assistance in running subjects. Address correspondence to M. S. Peterson, Department of Psychology, George Mason University, MS 3F5, 4400 University Dr., Fairfax, VA 22030 (e-mail: mpeters2@gmu.edu).

\section{REFERENCES}

Boot, W. R., Kramer, A. F., McCarley, J. S., Peterson, M. S., \& Scialfa, C. T. (2004, April). Older adults display a larger memory span than younger adults in visual search. Paper presented at the Cognitive Aging Conference-2004, Atlanta.

Boot, W. R., McCarley, J. S., Kramer, A. F., \& Peterson, M. S. (2004). Automatic and intentional memory processes in visual search. Psychonomic Bulletin \& Review, 11, 854-861.

Castel, A. D., Pratt, J., \& Craik, F. I. M. (2003). The role of spatial working memory in inhibition of return: Evidence from divided attention tasks. Perception \& Psychophysics, 65, 970-981.

Chun, M. M., \& JIANG, Y. (1998). Contextual cueing: Implicit learning and memory of visual context guides spatial attention. Cognitive Psychology, 36, 28-71.

DeUbEL, H., \& SCHNEIDER,W. X. (1996). Saccade target selection and object recognition: Evidence for a common attentional mechanism. Vision Research, 36, 1827-1837.

FINDLAY, J. M. (1997). Saccade target selection during visual search. Vision Research, 37, 617-631.

GibSon, B. S., LI, L., Skow, E., Brown, K., \& Cooke, L. (2000). Searching for one versus two identical targets: When visual search has a memory. Psychological Science, 11, 324-327.

Gilchrist, I. D., \& HARVEY, M. (2000). Refixation frequency and memory mechanisms in visual search. Current Biology, 10, 1209-1212.

Gilchrist, I. D., \& Harvey, M. (2006). Evidence for a systematic component within scanpaths in visual search. Visual Cognition, 14, 704-715.

GREENWOOD, P. M., \& PARASURAMAN, R. (2004). The scaling of spatial attention in visual search and its modification in healthy aging. Perception \& Psychophysics, 66, 3-22.

HAN, S. H., \& KIM, M. S. (2004). Visual search does not remain efficient when executive working memory is working. Psychological Science, 15, 623-628.

Henderson, J. M., \& Hollingworth, A. (1999). The role of fixation position in detecting scene changes across saccades. Psychological Science, 10, 438-443.

HoffMAN, J. E., \& REISs, J. (2001, November). When is visual search "amnesic"? Paper presented at the 42nd Annual Meeting of the Psychonomic Society, Orlando, FL.

Hoffman, J. E., \& Subramaniam, B. (1995). The role of visual attention in saccadic eye movements. Perception \& Psychophysics, 57, 787-795.

Horowitz, T. S., Holcombe, A. O., Wolfe, J. M., Arsenio, H. C., \& DiMase, J. S. (2004). Attentional pursuit is faster than attentional saccade. Journal of Vision, 4, 585-603.

Horowitz, T. S., \& WoLFE, J. M. (1998). Visual search has no memory. Nature, 394, 575-577.

HoROWITZ, T. S., \& WoLfe, J. M. (2001). Search for multiple targets: Remember the targets, forget the search. Perception \& Psychophysics, 63, 272-285.

IRWIN, D. E., \& Brockmole, J. R. (2004). Suppressing where but not what: The effect of saccades on dorsal- and ventral-stream visual processing. Psychological Science, 15, 467-473.

IRWIN, D. E., \& GoRDON, R. D. (1998). Eye movements, attention, and trans-saccadic memory. Visual Cognition, 5, 127-155.

Jiang, Y., Olson, I. R., \& CHUN, M. M. (2000). Organization of visual short-term memory. Journal of Experimental Psychology: Learning, Memory, \& Cognition, 26, 683-702.

JiANG, Y., \& WAGNER, L. C. (2004). What is learned in spatial contextual cuing - configuration or individual locations? Perception \& Psychophysics, 66, 454-463.

KLEIN, R. M., \& MACINNES, W. J. (1999). Inhibition of return is a foraging facilitator in visual search. Psychological Science, 10, 346-352.

Klein, R. M., Shore, D. I., MacInnes, W. J., Matheson, W. R., \& CHRIsTIE, J. (1998). Remember that memoryless search theory? Well forget it! Unpublished manuscript.

Kowler, E., Anderson, E., Dosher, B., \& Blaser, E. (1995). The role of attention in the programming of saccades. Vision Research, 35, 1897-1916.

KRISTJANSSON, A. (2000). In search of remembrance: Evidence for memory in visual search. Psychological Science, 11, 328-332.

McCarley, J. S., Wang, R. F., Kramer, A. F., IrWin, D. E., \& PeterSON, M. S. (2003). How much memory does oculomotor search have? Psychological Science, 14, 422-426.

MotTer, B. C., \& BeLKY, E. J. (1998). The guidance of eye movements during active visual search. Vision Research, 38, 1805-1815.

Он, S.-H., \& KIм, M.-S. (2004). The role of spatial working memory in visual search efficiency. Psychonomic Bulletin \& Review, 11, 275-281.

Peterson, M. S., Boot, W. R., Kramer, A. F., \& McCarley, J. S. (2004). Landmarks help guide attention during visual search. Spatial Vision, 17, 497-510.

Peterson, M. S., \& Kramer, A. F. (2001). Contextual cueing reduces interference from task-irrelevant onset distractors. Visual Cognition, 8, 843-859.

Peterson, M. S., Kramer, A. F., \& Irwin, D. E. (2004). Covert shifts of attention precede involuntary eye movements. Perception \& Psychophysics, 66, 398-405.

Peterson, M. S., Kramer, A. F., Wang, R. F., Irwin, D. E., \& McCarLEY, J. S. (2001).Visual search has memory. Psychological Science, 12, 287-292.

Ross, J., Morrone, M. C., Goldberg, M. E., \& Burr, D. C. (2001). Changes in visual perception at the time of saccades. Trends in $\mathrm{Neu}$ rosciences, 24, 113-121.

SaPIR, A., Hayes, A., Henik, A., Danziger, S., \& Rafal, R. (2004). Parietal lobe lesions disrupt saccadic remapping of inhibitory location tagging. Journal of Cognitive Neuroscience, 16, 503-509.

Theeuwes, J., Godisn, R., \& PRATT, J. (2004). A new estimation of the duration of attentional dwell time. Psychonomic Bulletin \& Review, 11, 60-64.

THORNTON, I. M., \& Horowitz, T. S. (2004). The multi-item localization (MILO) task: Measuring the spatiotemporal context of vision for action. Perception \& Psychophysics, 66, 38-50.

Treisman, A., \& Gormican, S. (1988). Feature analysis in early vision: Evidence from search asymmetries. Psychological Review, 95, 15-48. 
von Mühlenen, A., Müller, H. J., \& Müller, D. (2003). Sit-andwait strategies in dynamic visual search. Psychological Science, 14, 309-314.

WOLFE, J. M. (1994). Guided Search 2.0: A revised model of visual search. Psychonomic Bulletin \& Review, 1, 202-238.

Wolfe, J. M., \& Horowitz, T. S. (2004). What attributes guide the deployment of visual attention and how do they do it? Nature Reviews Neuroscience, 5, 495-501.

Woodman, G. F., \& Luck, S. J. (2004). Visual search is slowed when visuospatial working memory is occupied. Psychonomic Bulletin \& Review, 11, 269-274.

ZeLINSKY, G. J., \& SheInberg, D. L. (1997). Eye movements during parallel-serial visual search. Journal of Experimental Psychology: Human Perception \& Performance, 23, 244-262.

ZINGALE, C. M., \& KoWLER, E. (1987). Planning sequences of saccades. Vision Research, 27, 1327-1341.

\section{NOTES}

1. Because the smaller set size was less likely than the larger set size to occupy all of the spaces over the course of the experiment, we would have had to drop 5 subjects because of empty cells. In addition, the larger set size provides for a greater range of visitation orders, potentially leading to a larger contrast between the static and the dynamic conditions.

2. Although the RT slopes for the dynamic displays are higher in Experiment 1 than in Experiment 2, the differing error rates, particularly the interaction with set size in Experiment 2, make comparing search slopes across experiments dangerous.

(Manuscript received April 18, 2005; revision accepted for publication April 4, 2006.) 